\title{
Equivalence of nonequilibrium ensembles in turbulence models
}

Article

Accepted Version

Biferale, L., Cencini, M., De Pietro, M., Gallavotti, G. and Lucarini, V. (2018) Equivalence of nonequilibrium ensembles in turbulence models. Physical Review E, 98 (1). 012202. ISSN 2470-0053 doi: https://doi.org/10.1103/PhysRevE.98.012202 Available at https://centaur.reading.ac.uk/77946/

It is advisable to refer to the publisher's version if you intend to cite from the work. See Guidance on citing.

To link to this article DOI: http://dx.doi.org/10.1103/PhysRevE.98.012202

Publisher: American Physical Society

All outputs in CentAUR are protected by Intellectual Property Rights law, including copyright law. Copyright and IPR is retained by the creators or other copyright holders. Terms and conditions for use of this material are defined in the End User Agreement.

\section{www.reading.ac.uk/centaur}

\section{CentAUR}

Central Archive at the University of Reading 
Reading's research outputs online 


\title{
Equivalence of Non-Equilibrium Ensembles in Turbulence Models
}

\author{
Luca Biferale, ${ }^{1}$ Massimo Cencini, ${ }^{2}$ Massimo De Pietro, ${ }^{1}$ Giovanni Gallavotti, ${ }^{3}$ and Valerio Lucarini ${ }^{4,5,6}$ \\ ${ }^{1}$ Dipartimento di Fisica and INFN, Università di Roma "Tor Vergata", Via Ricerca Scientifica 1, 00133 Roma, Italy \\ ${ }^{2}$ Istituto dei Sistemi Complessi, CNR, via dei Taurini 19, 00185 Rome, Italy and INFN "Tor Vergata" \\ ${ }^{3}$ INFN-Roma1 and Università "La Sapienza Roma, Italy \\ ${ }^{4}$ Department of Mathematics and Statistics, University of Reading, Reading, RG66AX, United Kingdom \\ ${ }^{5}$ Centre for the Mathematics of Planet Earth, University of Reading, Reading, RG66AX, United Kingdom \\ ${ }^{6}$ CEN, University of Hamburg, Hamburg, 20144, Germany
}

(Dated: April 18, 2018)

\begin{abstract}
Understanding under which conditions it is possible to construct equivalent ensembles is key to advancing our ability to connect microscopic and macroscopic properties of non-equilibrium statistical mechanics. In the case of fluid dynamical systems, a first issue is to test whether different models for viscosity lead to the same macroscopic properties of the fluid systems in different regimes. Such models include, besides the standard choice of constant viscosity, also cases where the time symmetry of the evolution equations is exactly preserved, as it must be in the corresponding microscopic systems, when available. Here a time-reversible dynamics is obtained by imposing the conservation of global observables. We test the equivalence of reversible and irreversible ensembles for the case of a multiscale shell model of turbulence. We verify that the equivalence is obeyed for the mean-values of macroscopic observables, up to an error that vanishes as the system becomes more and more chaotic.
\end{abstract}

\section{INTRODUCTION}

The macroscopic description of the dynamics of physical systems typically include forces that phenomenologically model the effect of molecular disordered motions, and are controlled by appropriate transport coefficients (such as viscosity, diffusivity etc.). A prominent example is given by the viscous term of the Navier-Stokes (NS) equations. Such forces break the time-reversibility, which is instead inherent to the microscopic dynamics. They are also responsible for the dissipation of energy, which allows for establishing a (non-equilibrium) statistically steady state when the system is externally driven.

In the context of molecular dynamics a similar role is played by thermostats. A body of numerical simulations from the ' 80 s till these days have shown that the nonequilibrium properties of systems composed by a large number of molecules (particles) are basically independent of the precise nature (reversible or not) of the model used for the thermostats [1. This suggests that something similar may apply to the macroscopic description of physical systems, as pioneered in simulations in [2] and conjectured, on more theoretical ground, about two decades ago in [3, 4. Specifically the hypothesis is that the statistical properties of the non-equilibrium steady state of a macroscopic system, whose dynamics obeys a simple phenomenological law of the kind described above, should be equivalently described by different macroscopic equations, including some that preserve time reversal symmetry. In particular, with the example of fluid dynamics in mind, this can be realized by allowing the viscosity to depend on the fluid velocity in an appropriate way, thus converting the (inherently irreversible) dynamical ensemble of the Navier-Stokes equation with a fixed viscosity into a (formally reversible) dynamical ensemble with fluctuating viscosity. In systems at equilibrium, a conceptually similar step is done when switching from the microcanonical to the canonical ensemble.

The equivalence discussed above has already been scrutinized in a few simple systems such as a highly truncated version of the 2D Navier Stokes equations with periodic boundary conditions [5, 6] and, more recently, in the Lorenz '96 model [7. Such tests dealt with systems not exhibiting the time-scale separation typical of many macroscopic systems. In this paper we explore whether it is possible, and under which caveats, to establish an equivalence between different non-equilibrium ensembles in systems with multiple spatial and temporal scales. In particular, we investigate the shell model for turbulence introduced in [8] (see also [9, 10] for general surveys on shell models).

The study of multiscale systems is at the core of many disciplines dealing with complex systems and the construction of accurate methods for model reduction is of great relevance for the theory and for the construction of efficient and robust numerical models. For instance a substantial part of the efforts in weather and climate modeling are devoted to improving the representation of small scale processes. This requires a difficult interplay between Large Eddy Simulations (LES) [1, 12 and dedicated observational campaigns. LES simulations themselves need to be tailored via parametrization (which amounts to defining suitable eddy viscosities) to be compatible with higher resolution direct numerical simulations (DNS) of atmospheric flows. It is important to remark that reversible models for the dissipative terms are already used in LES [13] (see also [14, 15] for interesting studies on the interplay of energy cascade and reversibility in LES and DNS of the Navier-Stokes equations).

The paper is structured as follows. In Sect. II we provide a concise, but self-contained, summary of the general framework of non-equilibrium dynamical ensem- 
ble equivalence, where we formalize a general theoretical approach to the problem in the form of conjectures that can be subjected to tests.

In Sect. III we present the multiscale model analyzed in detail in this study. We supplement the traditional irreversible model with the reversible models obtained by replacing viscous terms with forces imposing anholonomous constraints on suitably chosen observables selected so that the resulting equations are time reversible.

Comparisons between properties of the irreversible and reversible models are discussed in Sect. IV] where we analyze a range of mathematical and physical properties of the models and assess whether the equivalence discussed above holds.

In Sect. V we summarize and discuss the main findings of our paper and present perspectives for future works in this direction.

\section{THE GENERAL FRAMEWORK: EQUIVALENCE OF ENSEMBLES}

\section{A. Equivalence of Equilibrium Ensembles}

One of the cornerstones of equilibrium statistical mechanics is the possibility of establishing an equivalence between different statistical ensembles [16, 17]. This means that in the thermodynamic limit - as the number of particles goes to infinity - the expectation values of physical observables of the system do not depend on the specific choice of the thermostat defining the interaction between the system and the reservoir it is in contact with, when suitable consistency is imposed.

Clearly not all physical observables will have the same value in the different ensembles. For instance, in a system statistically described by the canonical ensemble the temperature fluctuations vanish while energy fluctuates and the opposite occurs in a system described by the microcanonical ensemble.

Equivalence of equilibrium ensembles allows us to understand the emergence of macroscopic thermodynamical properties that do not depend on the details of the microscopic dynamics describing the coupling between a system and the surrounding environment.

\section{B. Equivalence of Non-Equilibrium Ensembles}

\section{General Discussion}

Let us consider the simplest case of an out-ofequilibrium system modeled by a differential equation with $N$ variables that can be thought as a time reversible equation perturbed with an external force, which injects energy into the system, plus a dissipative force, which absorbs energy, allowing the system to reach a steady state.
The parameter controlling dissipation (e.g. viscosity $\nu$ in a fluid) can be replaced by a multiplier defined in such a way that the new equation admits a suitably selected observable as an exact constant of motion (e.g. the fluid enstrophy). We will call it the balancing observable.

Furthermore the multiplier can often be chosen so that the new equations exhibit a time reversal symmetry (see below for typical examples). The multiplier will fluctuate in time and, for macroscopic observables, equivalence is expected between the irreversible and reversible formulations. By macroscopic we mean observables that depend on a few $(\ll N)$ large-scale degrees of freedom (hence insensitive to the details of the system when $N$ is large). One expects the equivalence to hold when the motion is sufficiently chaotic and the fluctuating multiplier has average equal to the value of the phenomenological dissipation parameter.

For instance, in the case of a fluid described by the incompressible Navier-Stokes equations in a homogeneous geometry (e.g. periodic boundary conditions) or by a shell model truncated at $N$ modes we have a multi-scale non equilibrium system characterized by a single dynamical parameter $R$, the Reynolds number, and an ultraviolet cutoff $N$. At fixed forcing, equivalence of the averages of a prefixed number of observable is expected in the limit of very small dissipation e.g. $\nu \rightarrow 0$ or, equivalently, Reynolds number $R \rightarrow \infty$. The discrepancy between averages of the prefixed observables is expected to become smaller than some $\delta>0$ for a $R$ above a threshold value $R_{\delta}$. In applications to fluids it is also expected that $N$ should be taken large enough and correspondingly the equivalence threshold will have to become $R>R_{\delta, N}$, i.e. depending on $N$ too. The order of the limit $R \rightarrow \infty$ and $N \rightarrow \infty$ is a delicate issue that will be discussed in the following for the specific case of the shell model.

Then the analogy with the usual theory of ensemble equivalence for equilibrium statistical mechanics would be complete with $\nu$, or the Reynolds number $R \propto \nu^{-1}$, playing the role of the inverse temperature, and with $N$ (necessary, perhaps, to give mathematical wellposedness to the equations in 3 dimensions or, certainly, in numerical implementations in any dimension) playing the role of the volume.

Of course, an important question is: how do we choose the balancing observable in order to successfully define an equivalent ensemble? For instance, in the NS case, the balancing can be constructed using the total enstrophy, or the total energy, or other macroscopic observables. The choice might be critical because the Fourier components of the velocity field have non-local interactions [18, so that the equivalence could be affected by the same difficulties that occur in equilibrium statistical mechanics in systems with long range interactions [19]. As far as we know, there is no general prescription and, in the end, the choice might be based on empirical grounds or motivated by/targeted to specific applications. 


\section{Mathematical Formulation}

Mathematically speaking, we consider a dynamical system with $N$ degrees of freedom written as:

$$
\dot{x}_{j}=f_{j}(x)+F_{j}-\nu(\mathbb{L} x)_{j}, \quad j=1, \ldots, N
$$

where $F_{j}$ is a constant forcing, $\nu>0$ is a dissipation coefficient and $\mathbb{L}$ is a positive definite dissipation matrix. In many interesting cases one has $(\mathbb{L} x)_{j}=g_{j} x_{j}$ with $g_{j}>0$ (the matrix is diagonal and all the elements on the diagonal are positive; no summation implied here).

A system is said to have a time reversal symmetry $I$ if the map $I$ acts on the variable $x$ so that if $t \rightarrow S_{t} x$ is a solution then $I S_{t} x=S_{-t} I x$, i.e. if $t \rightarrow x(t)$ is a solution also $I x(-t)$ is solution (with datum $I x(0)$ ). Therefore the above Eq. (1) has the map $I x=-x$ as a time reversal symmetry if $f_{j}(x)$ is even in $x$ and $\nu=0$.

Let $O(x)$ be an observable such that $\sum_{j=1}^{N} \partial_{j} O(x)(\mathbb{L} x)_{j}=M(x)$ is positive for $x \neq 0$. For instance in the above equation if $L=\nVdash$ and $O(x)=x^{2} / 2$ then $M(x)=x^{2}$. Then the equation:

$$
\dot{x}_{j}=f_{j}(x)+F_{j}-\alpha(x)(\mathbb{L} x)_{j},
$$

with

$$
\alpha(x) \equiv \frac{\sum_{j=1}^{N}\left(f_{j}+F_{j}\right) x_{j}}{M(x)}
$$

admits $O(x)$ as an exact constant of motion, i.e. $\dot{O}=0$. Furthermore, if $O(x)=O(-x)$, the equation is timereversible. In Eq. (1) the viscosity, $\nu$, is set constant and the observable $O$ fluctuates; correspondingly, in Eq. (2), the observable $O$ is constant and the "viscosity" $\alpha$ fluctuates.

Hereafter the notation $\left.X\right|_{y}$ will denote that $X$ is evaluated in the model where the quantity $y$ is kept constant. We say that the stationary distributions of the Eq. (1), (2) define ensembles of statistical distributions that can be parameterized by the value of $\nu$ for Eq. (1) or, by the (constant) value $\tilde{O}$ of the observable $O$ for Eq. (2).

In this work, equivalence means that the reflexivity property holds, i.e.

$$
\left.\langle O\rangle\right|_{\nu}=\left.\widetilde{O} \quad \leftrightarrow \quad\langle\alpha\rangle\right|_{\widetilde{O}}=\nu,
$$

and for a given set of macroscopic observables $\Phi$ the stationary averages in the reversible and irreversible evolutions are related by

$$
\left.\langle\Phi\rangle\right|_{\nu}=\left.\langle\Phi\rangle\right|_{\widetilde{O}}(1+o)
$$

with the $o$ a $\Phi$-dependent quantity, infinitesimal as $\nu^{-1} \rightarrow \infty$, for fixed $N$.

Equation (5) clarifies the thermodynamical aspect of the equivalence: in a strongly chaotic regime, measuring a macroscopic observable of the system, we are unable to say whether we are observing the reversible or the irreversible variant.
The property of reflexivity is an essential element of the proposed equivalence: setting the value of the viscosity coefficient $\nu$ in the irreversible system is conceptually equivalent to setting the value of the physical quantity $O$ in the corresponding reversible system.

The above-mentioned formulation of the equivalence conjecture has been extended in other studies $20-23$, including the definition of a fluctuation relation for the reversible ensemble, as well as conjectures about the equivalence of the Lyapunov spectra in the two ensembles 7 . However, these concepts are out of the scope of this paper, and will not be discussed in the following.

Finally, by repeating the procedure described above with a different observable $O$, it is possible to generate different time-reversible models, so that a plurality of (potentially equivalent) non-equilibrium ensembles can, in principle, be constructed.

\section{MODELS}

\section{A. The (irreversible) Shell Model}

Shell models are finite dimensional, chaotic dynamical systems providing a test bed for fundamental studies of fully developed turbulence $9,10,24$. They can be thought as drastic simplifications of the Navier-Stokes equations and share with them many non-trivial properties observed in experiments and simulations, such as the energy cascade from large to small scales, dissipative anomaly, and intermittency with anomalous scaling for the velocity statistics.

Our analysis is based on the shell model introduced in Ref. [8]. It describes the evolution of a set of complex variables $u_{n}$, representing the velocity in a shell of wavenumbers $|\mathbf{k}| \in\left[k_{n}, k_{n+1}\right]$, with $n=0, \ldots, N-1$. The Fourier shells $k_{n}$ are geometrically spaced, $k_{n}=k_{0} 2^{n}$ with $k_{0}=1$, so that a large, $O\left(2^{N}\right)$, range of scales can be explored using few degrees of freedom. The equations of motion take the form [8]:

$$
\dot{u}_{n}=\mathcal{N}\left[\left\{u_{n}\right\}\right]-\nu k_{n}^{2} u_{n}+F_{n}, \quad n=0, \ldots, N-1,
$$

where

$\mathcal{N}\left[\left\{u_{n}\right\}\right]=i k_{n}\left(2 a u_{n+2} u_{n+1}^{*}+b u_{n+1} u_{n-1}^{*}+\frac{c}{2} u_{n-1} u_{n-2}\right)$

accounts for the non-linear coupling between neighboring wave-numbers, $-\nu k_{n}^{2}$ is the dissipative term, and $F_{n}$ is an external force typically acting at large scales (here $F_{n}=F \delta_{n, 0}$, with $F$ constant). The boundary conditions $u_{-1}=u_{-2}=u_{N}=u_{N+1}=0$ are imposed.

Rigorous results 25] have been derived for equation [6], proving that it admits a unique global, regular solution for all initial data with finite enstrophy. Moreover, it has been shown that the attractor is finite dimensional with dimension not exceeding $\left(\log _{2} R\right)+\frac{1}{2} \log _{2}\left(\frac{13}{4} 3\right)$ (see Eq.(62) in Ref.[25]), and that the evolution of the shells 
$\leq K$ determines the evolution of the remaining modes if $K$ is large enough, i.e. larger than the Kolmogorov wavenumber (defined below).

When $\nu=F=0$, the model (6) has two quadratic invariants depending on the values of the $a, b, c$ parameters. The choice $a=1, b=-0.5$, and $c=0.5$ guarantees that the non-linear evolution (7) conserves the total energy (hereafter $\sum_{n}$ denotes the sum over all the shells)

$$
E=\sum_{n}\left|u_{n}\right|^{2}
$$

and the total helicity $H=\sum_{n}(-)^{n} k_{n}\left|u_{n}\right|^{2}$, as in the three-dimensional Navier-Stokes equations.

After multiplying Eq. (6) times $u_{n}^{*}$, adding the complex conjugate, and summing over all the shells from 0 to $M$, one obtains the equation for the time evolution for the energy contained in the first $M$ shells:

$$
\dot{E}_{M}=\Pi_{M}^{E}-2 \nu \sum_{n=0}^{M} k_{n}^{2}\left|u_{n}\right|^{2}+2 \sum_{n=0}^{M} \operatorname{Re}\left(F_{n} u_{n}^{*}\right)
$$

where

$$
\begin{aligned}
\Pi_{M}^{E}= & -2 k_{M}\left[2 a \Im\left(u_{M+2} u_{M+1}^{*} u_{M}^{*}\right)+\right. \\
& \left.+(a+b) \Im\left(u_{M+1} u_{M}^{*} u_{M-1}^{*}\right)\right]
\end{aligned}
$$

is the (instantaneous) energy flux through the $M^{\text {th }}$ shell.

The model given in eq. (6) spontaneously develops an energy cascade from the large (forced) scales to the small ones, with a constant energy flux at steady state. The energetics of such a system is given by eq. (9) with $M=N-1$, and reads as follows:

$$
\dot{E}=\epsilon-2 \nu \Omega
$$

where the rate of energy injection, $\epsilon=2 \sum_{n} \operatorname{Re}\left(F_{n} u_{n}^{*}\right)$, is bounded by $2|F| \sqrt{E}$, and $2 \nu \Omega$ is the rate of energy dissipation, with

$$
\Omega=\sum_{n} k_{n}^{2}\left|u_{n}\right|^{2}
$$

being the total enstrophy. The energy flux in 11 is equal to zero because the non linear term (7) conserves energy.

From Eq. (11) at stationary state and the Schwartz inequality, it follows that the average of $E$ is $\bar{E} \leq$ $|f|^{2}\left(k_{0} \nu\right)^{-2}$, implying the boundedness of the phase space asymptotically visited by the system.

At stationary state, realized when $\langle\epsilon\rangle=\langle 2 \nu \Omega\rangle$, the energy injected at large scales cascades towards the small scales with a constant flux, $\left\langle\Pi_{n}^{E}\right\rangle=-\langle\epsilon\rangle$, for all shells between the forcing one and the the Kolmogorov wavenumber $k_{\eta}=\langle\epsilon\rangle^{1 / 4} \nu^{-3 / 4}$, where dissipation becomes dominant over non-linear transfers (provided $k_{\eta}<k_{N-1}$ ) [9].

\section{B. A Class of Reversible Shell Models}

Following the procedure discussed in Sect. II (see also Refs. 3, 4]), we can define a class of time-reversible models out of Eq. (6) as

$$
\dot{u}_{n}=\mathcal{N}\left[\left\{u_{n}\right\}\right]-\alpha_{\chi}\left[\left\{u_{n}\right\}\right] k_{n}^{2} u_{n}+F_{n},
$$

where the fluctuating viscosity $\alpha_{\chi}$ is a function of the velocity variables, $\left\{u_{n}\right\}$, chosen such as to conserve a generic quadratic quantity of the form

$$
O_{\chi} \equiv \sum_{n} k_{n}^{\chi}\left|u_{n}\right|^{2}=\text { const }
$$

where the continuous parameter $\chi$ weighs differently the wavenumbers. With this choice, the fluctuating viscosity
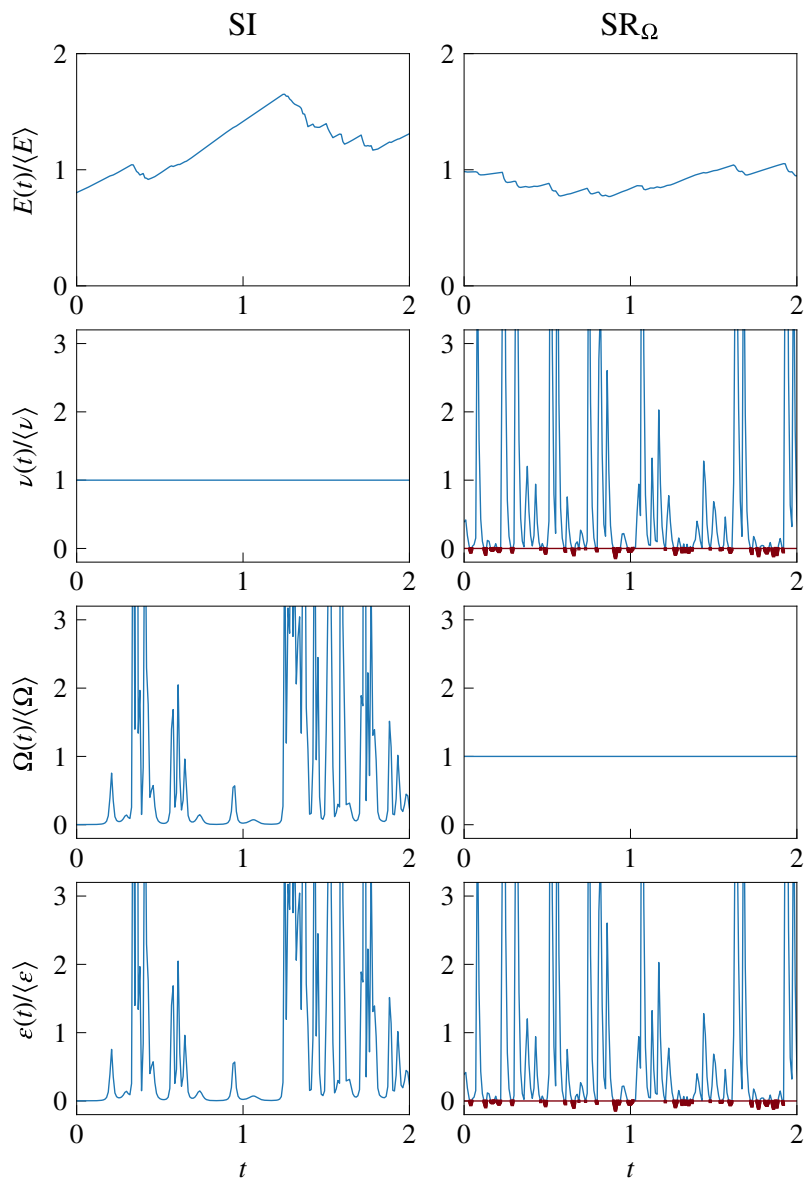

FIG. 1. (Color online) Side by side comparison between the time evolution of several observables in the irreversible shell model SI (left column) and the reversible $\mathrm{SR}_{\Omega}$ model (right column). From top to bottom: energy $E$, viscosity $\nu$ ( $\alpha_{2}$ for $\left.\mathrm{SR}_{\Omega}\right)$, enstrophy $\Omega$, and energy dissipation $\varepsilon=2 \nu \Omega\left(2 \alpha_{2} \Omega\right.$ for $\left.\mathrm{SR}_{\Omega}\right)$. All quantities are normalized by their average value. We used $N=15$ shells and $\nu=10^{-5}$, corresponding to the energy cascade regime. Notice that $\alpha_{2}$ is not positive definite: the occurrence of negative values, highlighted with a thicker/red line, corresponds to instances in which the dissipative terms injects energy into the system. 
takes the form

$$
\begin{aligned}
\alpha_{\chi}= & \frac{\sum_{n} k_{n}^{\chi \Re(}\left(u_{n} F_{n}^{*}\right)}{\sum_{n} k_{n}^{\chi+2}\left|u_{n}\right|^{2}}+ \\
& +\frac{\sum_{n} k_{n}^{\chi+1}\left(2 a C_{3, n+1}+b C_{3, n}-(c / 2) C_{3, n-1}\right)}{\sum_{n} k_{n}^{\chi+2}\left|u_{n}\right|^{2}}
\end{aligned}
$$

where $C_{3, n} \equiv-\Im\left(u_{n+1} u_{n}^{*} u_{n-1}^{*}\right)$. Notice that the constraint (14) implies that also the reversible models evolve in a bounded region of the phase-space.

For simplicity, we will denote the irreversible shell model SI and the reversible ones as $\mathrm{SR}_{\chi}$, where $\chi$ is the same parameter as in Eq. (14), representing the observable kept constant by the time-dependent viscosity. Two limiting cases of interest are $\chi=0$ and $\chi=2$, which we will also indicate as $\mathrm{SR}_{E}$ and $\mathrm{SR}_{\Omega}$, respectively.

The case $\chi=0$ corresponds to fixing the total energy $O_{\chi=0}=E$. Being the energy conserved in the inviscid limit, the second term on the right-hand side of 15 is zero. Notice also that while the constraint $\chi=0$ is apparently "democratic" on all wave-numbers, in the presence of an energy cascade it weighs more the first shells (large scales).

The second case corresponds to fixing the enstrophy (12), $O_{\chi=2}=\Omega$, which, due to the factor proportional to the square of the wave-number, puts most of the weight on the small scales. The limit $\chi=2$ is particularly interesting as the energy dissipation rate in the original model (6), $\varepsilon(t)=2 \nu \Omega$, fluctuates by virtue of the fluctuations of $\Omega$, while in the reversible model $(13) \varepsilon(t)=2 \alpha_{2} \Omega$ fluctuates with the viscosity $\alpha_{2}$. This phenomenology is clear from Fig. 1 where we present an overview of the dynamics of the standard SI model and the $\mathrm{SR}_{\Omega}(\chi=2)$ model. The figure shows the time evolution of some observables of interest such as $E, \Omega \nu$ and $\varepsilon$, in a situation of energy cascade for both the systems. These results will be analyzed in more detail in the next section.

We remark that some preliminary study of the model with $\chi=2$ was presented in Ref. [26], where the aim was not that of studying the equivalence in the sense specified in Sect. II.

\section{NUMERICAL SIMULATIONS}

\section{A. Setup of the Numerical Simulations}

We first integrate the irreversible (SI) model (6) for as long as needed to achieve stationarity. The resulting average energy spectrum $\left.\left.E_{n}\right|_{\nu} \equiv\left\langle\left|u_{n}\right|^{2}\right\rangle\right|_{\nu}$ is then used to set the initial condition for the reversible model $\sqrt{13}$ as

$$
\left.u_{n}(t=0)\right|_{\chi} \equiv \sqrt{\left.E_{n}\right|_{\nu}} e^{i \xi_{n}}
$$

where $\xi_{n}$ are random phases. The initial condition 16 guarantees that the reversible dynamics starts with initial values for the considered global quadratic observable
$O_{\chi}$ (see Eq. 14]), such as e.g. $E$ or $\Omega$, equal to the expectation value obtained with the irreversible model.

Simulations of SI are performed fixing the number of shells, holding constant the large scale forcing and varying the viscosity $\nu$ which plays here the role of the inverse of the Reynolds number $R=1 / \nu$. A change in the chosen value of the viscosity $\nu$ reflects in an initial configuration for the reversible models with different values of the conserved quantity $O_{\chi}$.

The corresponding reversible model is then integrated with the same number of shells $N$ and forcing $F_{n}$ as the irreversible case. As for the forcing, we have chosen a constant (hence, time-reversible) forcing acting on the first shell only, i.e. $F_{n}=\delta_{n, 0}|F| e^{i \gamma}$ with $|F|=1$ and $\gamma$ a randomly chosen phase.

A statistical ensemble of 10 dynamical evolutions was obtained by varying the phases $\xi_{n}$ of the initial condition. All data presented hereafter are averages on this ensemble and the errors are estimated as the standard error on the mean. The characteristic time of the large scales is estimated as $T_{L} \sim E /\langle\varepsilon\rangle$ which is $O(1)$ in our simulations. The total integration time (cumulated over all the simulations in the ensemble) ranges between $\sim 10^{5} T_{L}$, for the smallest Reynolds, and $\sim 10^{4} T_{L}$ for the largest.

The integration scheme was a modified $4^{\text {th }}$ order Runge-Kutta with explicit integration of the linear part (see Appendix A for details). For both the irreversible and reversible models, the (fixed) integration time step was guaranteed to be $\delta t \leq \tau_{\min } / 50$, with $\tau_{\min }=$ $\min _{n}\left(\left\langle\left|u_{n}\right|\right\rangle k_{n}\right)^{-1}$ being the fastest time scale of the dynamics. The number of shells in the system was $N=20$, unless otherwise specified.

\section{B. Test of the equivalence in the reversible model conserving the total enstrophy}

We start by discussing the reversible model $\mathrm{SR}_{\Omega}$ obtained by imposing the conservation of enstrophy. Reversible models conserving other quadratic quantities (in particular, $\mathrm{SR}_{E}$ which conserves energy) will be discussed in the next section.

Phenomenology of the irreversible model: First it is useful to illustrate briefly the phenomenology of the irreversible model with fixed number of shells at increasing the Reynolds number, viz. decreasing the viscosity value $\nu$. In Figure 2, we show the energy spectrum obtained for three values of $\nu$. When the viscosity is small enough but such that $k_{\eta} \ll k_{N-1}$ (i.e. when the dissipative scale is well resolved), the irreversible shell model develops an energy cascade, from large to small scales, with a characteristic Kolmogorov-like scaling, $E_{n}=\left\langle\left|u_{n}\right|^{2}\right\rangle \propto k_{n}^{-2 / 3}$, plus intermittency corrections [9]. This energy cascade regime is well evident for $\nu=10^{-6}$ in Fig. 2. When the Reynolds number is very large, viz. the viscosity is so small that $k_{\eta} \gg k_{N-1}$, a new stationary regime sets in. In the following such regime will be referred as quasi-equilibrium as it is characterized by the energy be- 
ing essentially equipartited - though in non-equilibrium conditions - among the shells (see the case $\nu=10^{-12}$ in Fig. 2p, and by an average energy flux constant over the shells and typically much smaller than its fluctuations (not shown). The transition between the energy-cascade and quasi-equilibrium regimes is characterized by energy spectra with intermediate characteristics (see the case $\nu=10^{-10}$ in Fig. 2). Strictly speaking, the dynamical equivalence discussed in Sect. IIB 2 is expected to hold in the quasi-equilibrium regimes, when the Reynolds number $R \sim \nu^{-1}$ is large enough and $N$ is fixed. Note that in 7 the validity of the equivalence has been confirmed exactly in such quasi-equilibrium conditions.

Test of the Equivalence Conjecture: As a preliminary test of the equivalence, we first verified the validity of Eq. (4), i.e.. we checked whether the average value $\left\langle\alpha_{2}\right\rangle$ measured in the $\mathrm{SR}_{\Omega}$ model simulations converges to the values of the viscosity $\nu$ of the corresponding irreversible model. In Figure 3 we show the ratio $\left\langle\alpha_{2}\right\rangle / \nu$ at varying $R\left(R=\nu^{-1}\right.$, where $\nu$ is the viscosity of the SI model $)$. As one can see the ratio is $\approx 1$ for $R>10^{-5}$ and unity is approached better and better at increasing $R$, apart from the highest $R$, where computational constraints on the integration time leads to poorer convergence of the statistics and thus larger statistical errors.

As discussed in Sect. II B 2, the validity of Eq. (4) is a prerequisite for the equivalence conjecture. Then, we tested the conjecture (5) at varying values of $R$ using as observable $\Phi$ the $2^{\text {nd }}$ and $4^{\text {th }}$ moments of $\left|u_{n}\right|$ for a small wavenumber (shell $n=2$, see Fig. 4a). These moments are effectively large scales observables, and the equivalence conjecture is expected to hold for them for high values of $R$. We also measured the same moments at a larger wavenumber (shell $n=10$, see Fig. $4 \mathrm{~b}$ ) where, in principle, the validity of the equivalence should not be taken for granted. At large scales (Fig. 4 4) the data

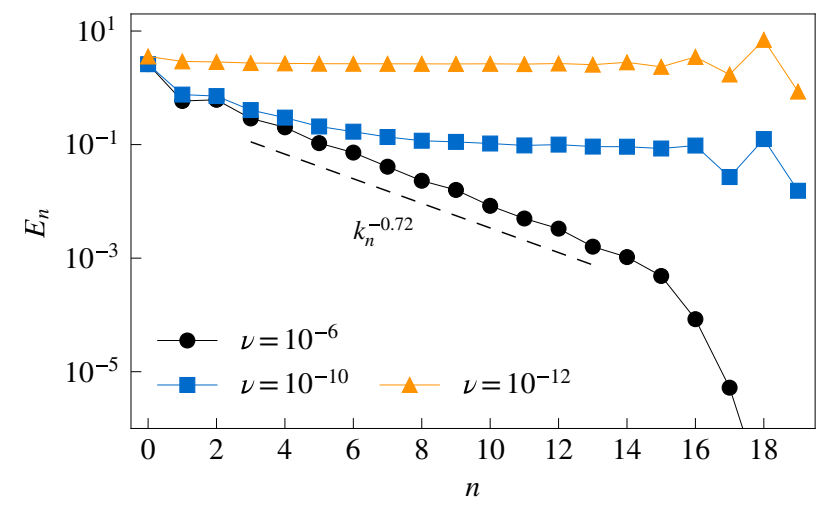

FIG. 2. (Color online) Phenomenology of the irreversible shell model. Energy spectrum $E_{n}=\left\langle\left|u_{n}\right|^{2}\right\rangle$ for three values of the viscosity $\nu$ with $N=20$ shells. With $\nu=10^{-6}$ the characteristic spectrum of the energy cascade $E_{n} \sim k_{n}^{-0.72}$ (see dashed line) appears. For $\nu=10^{-12}$ the energy spectrum is roughly at equipartition, namely the regime of quasiequilibrium (see main text). For $\nu=10^{-10}$ a mixed behavior is observed.

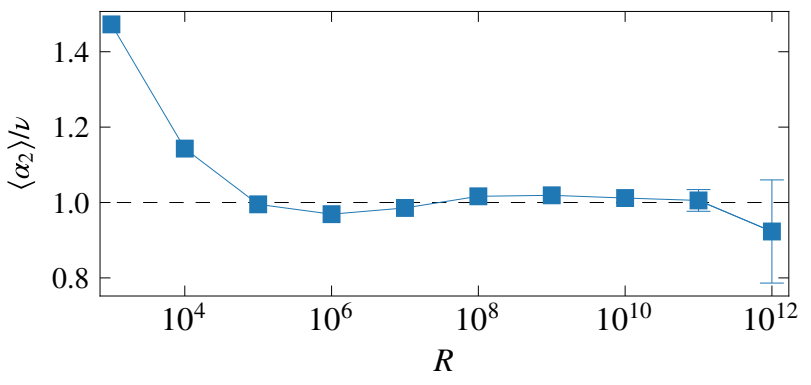

FIG. 3. Mean values of $\alpha_{2} / \nu$ for simulations of the $\mathrm{SR}_{\Omega}$ model at different Reynolds number $R$ with $N=20$ shells. The $R$ dependency of the $\mathrm{SR}_{\Omega}$ model is intended in the sense that it is initialized with an initial enstrophy $\tilde{\Omega}$ equal to $\langle\Omega\rangle$ measured in a run of the SI model with (fixed) viscosity $\nu=$ $R^{-1}$. The large error bars reported for high $R$ can be ascribed to the limited statistics, due to the high cost of the numerical integration in that range of parameters.
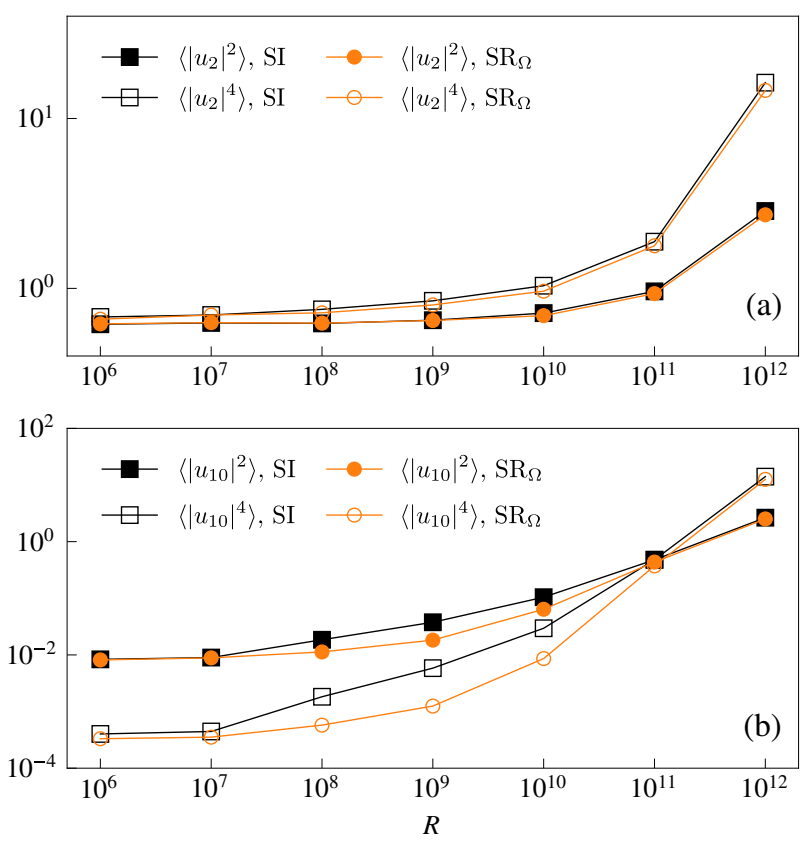

FIG. 4. (Color online) Test of the equivalence for the $\mathrm{SR}_{\Omega}$ model. $2^{\text {nd }}$ moment (full symbols) and $4^{\text {th }}$ moment (empty symbols) of a velocity field component pertaining to the large scales $n=2$ (a) and small scale $n=10$ (b), as functions of the Reynolds number $R$, for both the SI and $\mathrm{SR}_{\Omega}$ models with $N=20$ shells. The $R$ dependency of the $\mathrm{SR}_{\Omega}$ model is intended in the sense that it is initialized with an initial enstrophy $\tilde{\Omega}$ equal to $\langle\Omega\rangle$ measured in a run of the SI model with (fixed) viscosity $\nu=R^{-1}$. Errors are smaller than or of the order of the symbol size.

points of the $\mathrm{SR}_{\Omega}$ perfectly agree with the values of the SI model at all the $R$ considered. At smaller scales (Fig. 4b) we observe a good agreement with SI at high and relatively small $R$, i.e. in both the quasi-equilibrium and energy-cascade regime, while deviations are present at intermediate values of $R$.

In order to understand better the above findings, in 

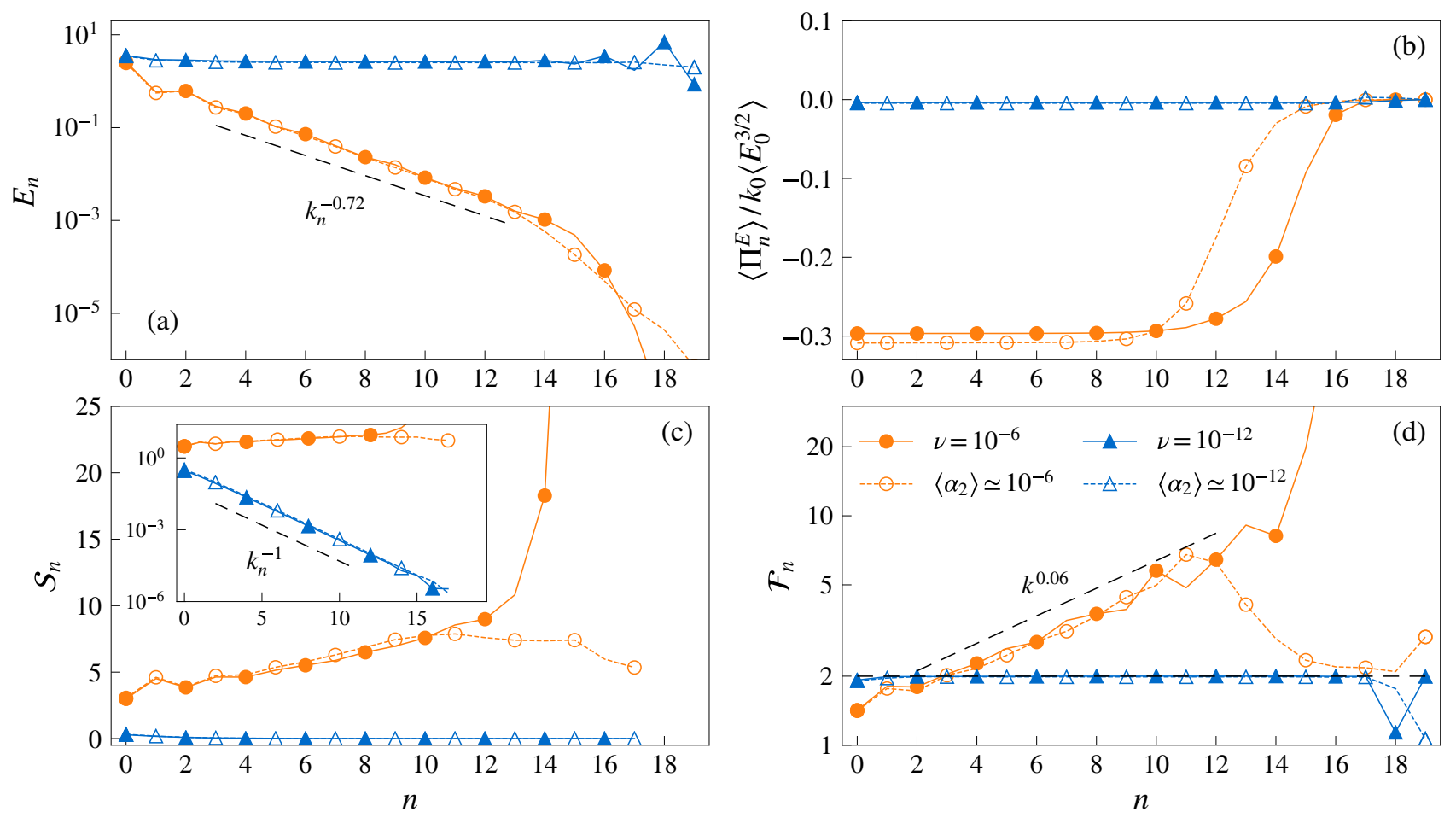

FIG. 5. (Color online) Comparison of several spectral observables between the SI and the SR $\Omega$ models, in both situations of energy cascade $\left(\nu=10^{-6} \bullet,\left\langle\alpha_{2}\right\rangle \simeq 10^{6} \circ\right)$ and quasi-equilibrium $\left(\nu=10^{-12} \Delta,\left\langle\alpha_{2}\right\rangle \simeq 10^{12} \triangle\right)$. Panels refer to: (a) Energy spectra; (b) energy flux (10); (c) skewness $\mathcal{S}_{n}$ (17); in the inset the same plot with logarithmic y-axis; (d) flatness $\mathcal{F}_{n}$ (18). Errors are of the order or smaller than the symbol size. The dashed line labeled $k_{n}^{-0.72}$ in (a) represents the scaling behavior a lá Kolmogorov plus intermittency correction. The dashed line labeled $k_{n}^{-1}$ in the inset of panel (c) represents a dimensional prediction valid at quasi-equilibrium; indeed, since $\left\langle\left|u_{n}\right|\right\rangle$ and $\left\langle\Pi_{n}^{E}\right\rangle$ do not depend on the wavenumber $k_{n}$, at least in a certain range of scales, as shown in panel (a) and (b) respectively, one has that $\mathcal{S}_{n} \sim k_{n}^{-1} \sqrt{17}$. The dashed line labeled $k_{n}^{0.06}$ in panel (d) shows a best fit of the curves in the cascade regime. Finally, the horizontal dashed line in panel (d) displays the value $\mathcal{F}_{n}=2$, which is expected for complex Gaussian variables. In these figures, and in some of the following ones, to ease the identification of the various curves and avoid the superposition of different symbols, not all data points have been marked by a symbol.

Fig. $5 \mathrm{a}$ we compare the energy spectra of the SI and $\mathrm{SR}_{\Omega}$ in the two regimes of energy cascade and quasiequilibrium. Consistently with Fig. 4, a very good equivalence between the reversible and irreversible models is observed in both regimes, at least at large enough scales. At small scales deviations can be seen in both regimes.

In the cascade regime, the main differences appear for $k_{n}>k_{\eta}$. It should be noticed that $k_{\eta}>k_{10}$, which explains the agreement observed in Fig. 44. Clearly, choosing a wavenumber $k_{n}>k_{\eta}$ does lead in general to a good agreement. It is worth noticing that for $k_{n}>k_{\eta}$ the energy spectrum has a scaling law close to $E_{n} \sim k_{n}^{-2}$, which could be due to a local equipartition of the enstrophy (which is mostly localized around these scales) 27].

In the quasi-equilibrium regime, we can notice that the $\mathrm{SR}_{\Omega}$ model shows a more regular spectrum at small scales (near the boundary $k_{N-1}$ ), with respect to the SI model. We should remark that these oscillations in the SI model remain confined to the last three/four shells, as confirmed by simulations with a larger number of shells (not shown). Our interpretation is that they are simply due to the constraint imposed by the fixed ultraviolet cut- off, that becomes important when the scales affected are not efficiently damped by viscosity. The choice $\Omega=$ const imposes a constraint on the amount of energy present at scales around $k \sim \sqrt{\Omega / E}$, suppressing such oscillations coming from the spectral truncation.

We also compared other quantities in the two models at varying the Reynolds number. In particular, we studied the average energy flux (10) (Fig. 5b), the skewness (Fig. 5c) defined as

$$
\mathcal{S}_{n}=\frac{\left\langle\Pi_{n}^{E}\right\rangle}{k_{n}\left\langle\left|u_{n}\right|\right\rangle\left\langle\left|u_{n+1}\right|\right\rangle\left\langle\left|u_{n+2}\right|\right\rangle},
$$

where we use products $\left|u_{n}\right|\left|u_{n+1}\right|\left|u_{n+2}\right|$ in place of $\left|u_{n}\right|^{3}$ to get rid of spurious oscillations due to the phase symmetry between three adjacent shells (see 8 for details), and the flatness (Fig. 5d)

$$
\mathcal{F}_{n}=\frac{\left\langle\left|u_{n}\right|^{4}\right\rangle}{\left\langle\left|u_{n}\right|^{2}\right\rangle^{2}} .
$$

In the cascade regime, the equivalence holds only within the inertial range of scales, which is slightly shorter in 
the $\mathrm{SR}_{\Omega}$ compared to SI, indeed, as clear from Fig. 5 , the flux for $\mathrm{SR}_{\Omega}$ model stops to be constant at slightly smaller wavenumbers than in the SI model. We observe a remarkable agreement also for very delicate properties such as the intermittent corrections to the scaling exponents as clear from both the energy spectrum (Fig. 5a) and high order quantities such as $\mathcal{S}_{n}$ and $\mathcal{F}_{n}$ (Fig. 5 F,d). A previous study confirmed this equivalence also on higher order structure functions $\left\langle\left|u_{n}\right|^{q}\right\rangle$, up to order $q=9$ [26]. These results offer further confirmation of the extreme robustness of the energy cascade mechanism with respect to the particular method used to remove energy at small scales, thus reinforcing the validity of the dynamical equivalence.

Also in the quasi-equilibrium regime (i.e. for the simulation corresponding to $\nu=10^{-12}$ ) a very good equivalence is observed for all the quantities. In particular, we notice that in the quasi-equilibrium regime the statistics tends to become Gaussian with $\mathcal{S}_{n} \rightarrow 0$ and $\mathcal{F}_{n} \approx 2$ (which is the result expected for Gaussian statistics, taking into account the fact that $u_{n}$ is complex).

Between these two regimes, for intermediate values of the viscosity, deviations are well evident [as already clear from Fig. (4)b].

Summarizing, the equivalence conjecture is well verified in the quasi-equilibrium regime, where is expected to hold, at almost all scales excluding those very close to the ultraviolet cut-off. Remarkably, the equivalence holds, even for very delicate quantities, also in the energycascade regime at scales $k_{n} \lesssim k_{\eta}$. We notice that the equivalence in the latter case may have a different nature from that of the former. In particular, when the energy cascade is at play, the matching of the statistics of the various observables within the inertial range may be due to the robustness of the inertial range physics with respect to the energy removing mechanisms, i.e. due to the dissipative anomaly.

\section{Test of the Equivalence in Reversible Models Conserving Different Quantities}

Here, we discuss the equivalence in the reversible models (13) at varying the parameter $\chi$ in (14), i.e. at varying the particular quadratic quantity conserved by the timedependent viscosity.

We start from Fig. 6 that, analogously to Fig. 4, shows the $R$ dependence of the 2 nd and 4 th moment of $\left|u_{n}\right|$ for $n=2$ (panel a) and $n=10$ (panel b) for the model $\mathrm{SR}_{E}$, i.e. when the reversible model is obtained by imposing the conservation of energy. Unlike the $\mathrm{SR}_{\Omega}$ model shown in Fig. 4. we can see that agreement between the moments of the SI and $\mathrm{SR}_{E}$ models is realized only in the quasi-equilibrium regime. This is further confirmed in Fig. 7 where we compare the energy spectra of the $\mathrm{SI}$ and $\mathrm{SR}_{E}$ models in this regime. As clear from the figure, for model $\mathrm{SR}_{E}$ the agreement of the spectra extends even close to the ultraviolet cut-off (compare with
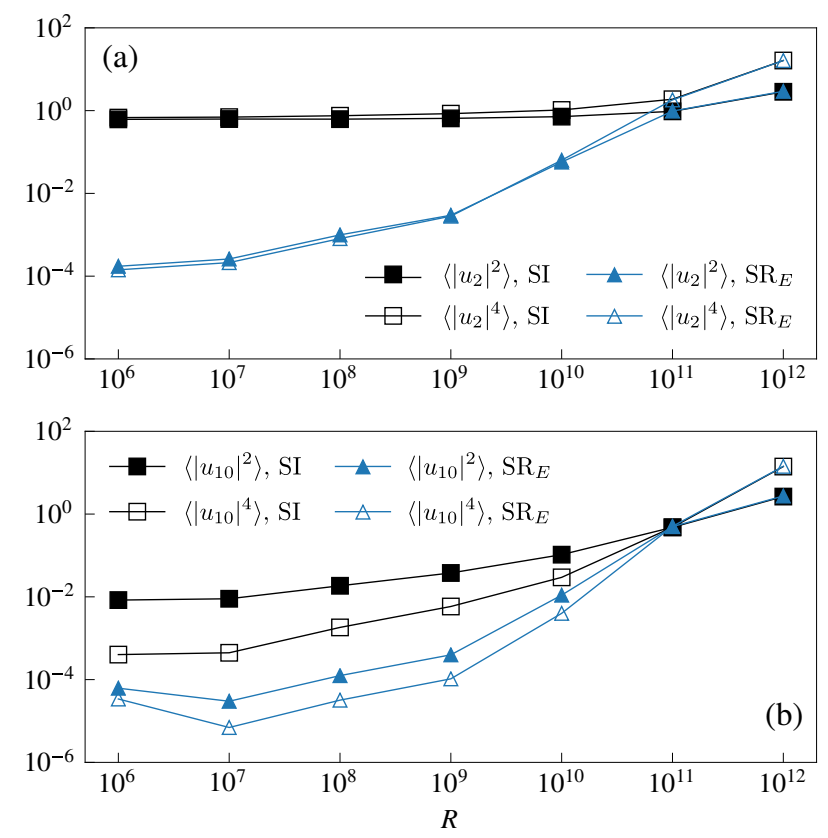

FIG. 6. (Color online) Test of the equivalence for the $\mathrm{SR}_{E}$ model. 2nd (full symbols) and 4th moment (empty symbols) of a velocity component at large scales $n=2$ (a) and small scales $n=10$ (b) as functions of the effective Reynolds number $R$, for the SI and $\mathrm{SR}_{E}$ models with $N=20$ shells. The $R$ dependency of the $\mathrm{SR}_{E}$ model is intended in the sense that it is initialized with an initial energy $\tilde{E}$ equal to $\langle E\rangle$ measured in a run of the SI model with (fixed) viscosity $\nu=R^{-1}$. Errors are smaller than or of the order of the symbol size.

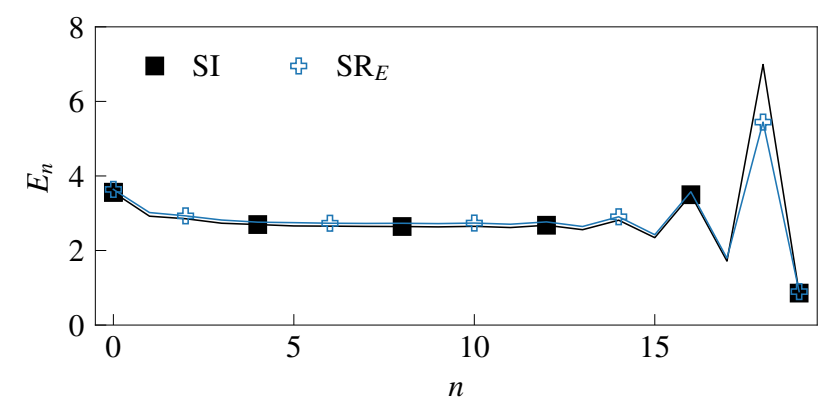

FIG. 7. (Color online) Energy spectra $E_{n}$ of the SI and $\mathrm{SR}_{E}$ models in the regime of quasi-equilibrium $(N=20, \nu=$ $\left.10^{-12}\right)$. Error bars are smaller than or of the order of the symbol size.

Fig. 5a). This is possibly due to the fact that the constraint of constant energy is less stringent for the large wave numbers compared with constant enstrophy constraint.

In order to understand the large differences between the $\mathrm{SR}_{E}$ and SI model out of the quasi-equilibrium regime we now fix $\nu$ such that the SI model is in the energy cascade regime. In Figure 8 8 we show the spectra obtained for different reversible models, all initialized with the same initial condition, conserving quadratic quantities $O_{\chi}$ indexed by different values of $\chi$ as from 


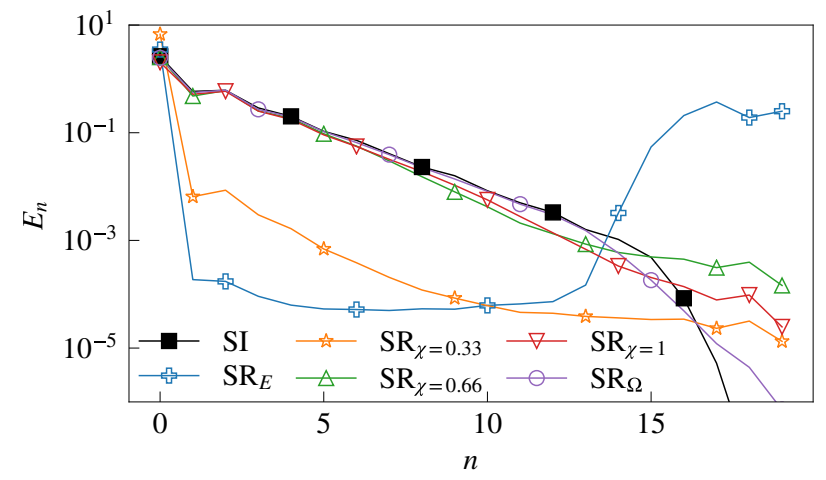

FIG. 8. (Color online) Energy spectra $E_{n}$ for several reversible models, compared with the irreversible one ( $\mathbf{\square}$, with $N=20$ and $\nu=10^{-6}$ ). All the reversible model simulations are initialized with the same distribution of initial energy in the range $0 \leq n<15$, but the models conserve different invariants $O_{\chi}$ [see eq. [14]]. Error bars are smaller than or of the order of the symbol size.

Eq. (14) (we recall that $\mathrm{SR}_{E}$ corresponds to the case $\chi=0)$. We see that there is a clear trend of better and better equivalence at increasing $\chi$, i.e. when the constraint weighs more and more the small scales. In particular, when the reversible model conserves $O_{\chi}$ with low values of $\chi$, it suffers from the lack of a stable energy cascade solution, with the effective confinement of the dynamics on the shell $n=0$. When the value $\chi$ is large, on the contrary, $O_{\chi}$ is significantly dependent on the small scales of the system, meaning that the request $O_{\chi}=$ const actually imposes a constraint on the amount of energy needed in the small scales, favoring the presence of a stable energy cascade mechanism. The threshold between the two cases lies around $\chi=2 / 3$. Even if we did not pursued a systematic test, here is a simple argument for why the value $\chi=2 / 3$ should be a good candidate for the threshold: for that value both the constant energy flux solution and the $O_{\chi}$ equipartition solution have the same spectral scaling $E_{n} \sim k_{n}^{-2 / 3}$. For $\chi>2 / 3$ the constant energy flux solution has a less steep energy spectrum and it is likely dominant in the dynamics, and vice versa. Thus, given the same initial conditions for the velocity field, the model $\mathrm{SR}_{\Omega}$ and the other SR models with $\chi>2 / 3$ are always able to reach a chaotic stationary state with an energy cascade like the SI model.

Instead, in the same range of viscosities, the $\mathrm{SR}_{E}$ model and the other SR models with $\chi<2 / 3$ get locked in a fixed point in phase space, where all the energy of the system is localized in the $n=0$ shell and $\alpha_{0} \sim 1$.

The presence of an attractive fixed point in a highly dimensional phase-space unavoidably makes the statistical properties strongly sensitive to the extension in time of the dynamical evolution and to the total number of degrees of freedom. For example, we found that the results published in 28 were affected by the limited extension of the time integration and that by averaging more, as it is possible with the nowadays computational power, the long-time asymptotic dynamics is always dominated by the fixed point at small shell numbers.

Although we did not perform systematic tests, on the basis of the previous observations and of Fig. 7, it is reasonable to expect that for any $\chi$ equivalence should hold in the quasi-equilibrium regime.

Specifically, for the model $\mathrm{SR}_{E}$, it is worth remarking that imposing the conservation of energy constrains the energy dissipation to be identical to the energy input at any instant. This is at odds with the phenomenology of the cascade where such a balance is obtained only on average. On the other hand, fixing $\Omega=$ const does not introduce such stringent conditions on the instantaneous energy budget. More importantly, while the energy input varies on the (slow) time scale typical of the large scales the energy dissipation has a fast evolution. Thus, the model $\mathrm{SR}_{E}$ imposes a very severe dynamical constraint requiring the two quantities to be identical at each time. This constraint is less stringent in quasi-equilibrium conditions, where energy is essentially in equipartition among the shells. Indeed in such a regime also model $\mathrm{SR}_{E}$ becomes equivalent to the $\mathrm{SI}$ as clear from Fig. 7 .

\section{Analysis of the Time-Dependent Viscosity in the reversible model with enstrophy conservation}

In this section we study the statistics of the timedependent viscosity $\alpha_{2}$ in the $\mathrm{SR}_{\Omega}$ model. We have already shown that $\left\langle\alpha_{2}\right\rangle \approx \nu$ (Fig. 3) as required for the validity of the equivalence. However, the temporal fluctuations of $\alpha_{2}$ are non-trivial: as shown in Fig. $11 \alpha_{2}$ can become negative (i.e. the viscous forces can inject energy instead of removing it), which is the signature of the dynamical reversibility. In this section, though this is not directly linked with testing the equivalence conjecture, we explore how the statistics of this sign variations depends on the Reynolds number.

In Figure 9 we summarize the behavior of the timedependent viscosity $\alpha_{2}$ in different regimes: from quasiequilibrium to energy cascade (as qualified by the behavior of the spectra shown on the right panels). On the left panels we show the time evolution of $\alpha_{2}$ in a typical run of the model, in the central panel the measured PDFs of the values of $\alpha_{2}$, and on the right column the energy spectrum of the corresponding simulation. All data refer to the $\mathrm{SR}_{\Omega}$ model.

In the quasi-equilibrium regime, the viscosity $\alpha_{2}$ tends to have a PDF symmetric around the zero, becoming more and more skewed towards positive values as the cascade regime becomes dominant in the dynamics. A similar behavior of the PDF of the time-dependent viscosity of the reversible model as a function of the Reynolds number was found in [7]. In the limit of an extremely well resolved system $(N \rightarrow \infty$, with finite Reynolds number, i.e. in the energy-cascade regime with well resolved dissi- 


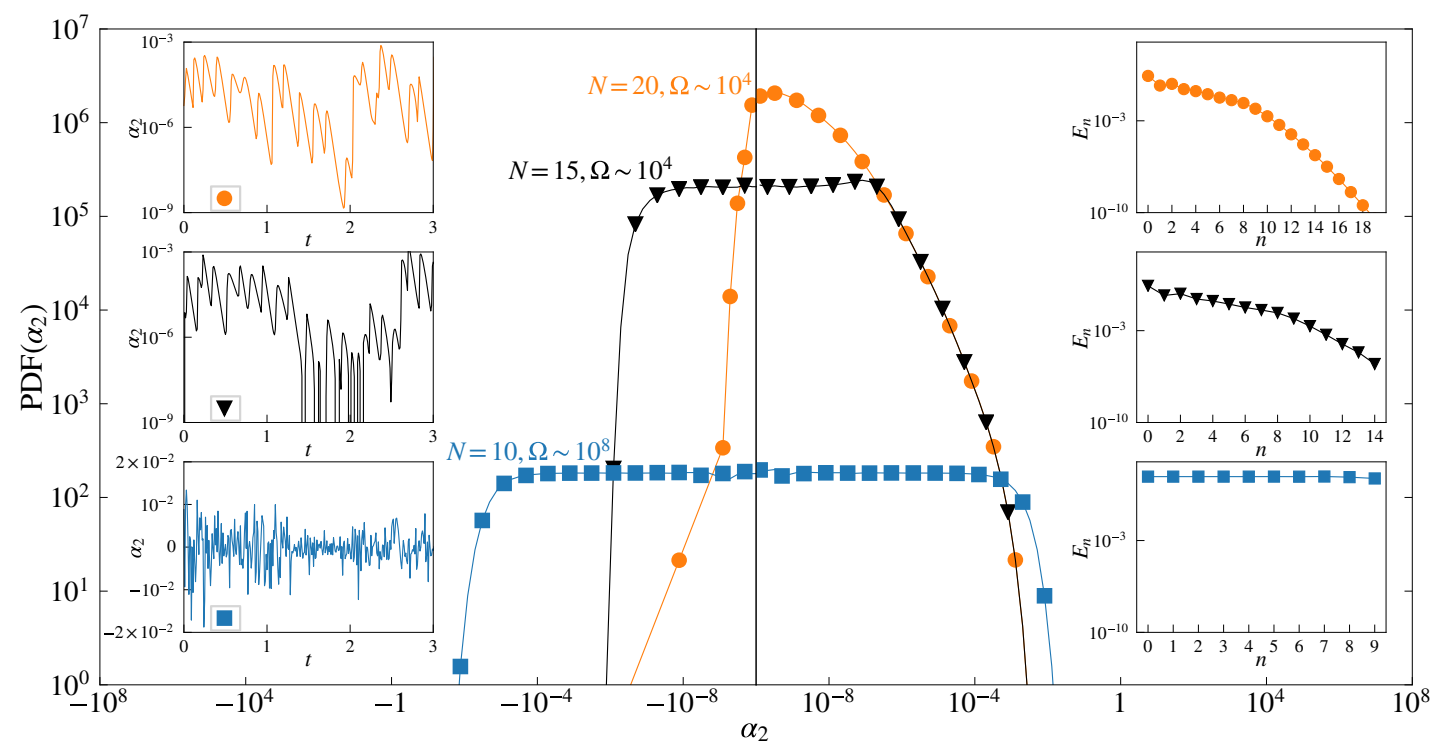

FIG. 9. (Color online) Probability Density Function of the time-dependent viscosity $\alpha_{2}$ for model SR $\Omega$ in three different cases: a situation of energy cascade $\left(N=20\right.$ and $\left.\Omega \sim 10^{4}, \bullet\right)$, a situation of quasi-equilibrium $\left(N=10\right.$ and $\left.\Omega \sim 10^{8}, \square\right)$, and a case in between $\left(N=15\right.$ and $\left.\Omega \sim 10^{4}, \boldsymbol{\nabla}\right)$. The insets on the left show corresponding typical time evolutions of $\alpha_{2}$. The insets on the right show the corresponding energy spectra.

pative range), the probability to observe negative values $\left(\alpha_{2}<0\right)$ within the observation time becomes extremely small. This observation shows once again the different nature of the equivalence in the quasi-equilibrium regime (corresponding to taking the limit $R \rightarrow \infty$ with $N$ fixed, eventually very large) and the cascade one (corresponding to taking the limit $N \rightarrow \infty$ with $R$ fixed and very large).

\section{CONCLUSIONS}

Summarizing, in this paper we have scrutinized the validity of the equivalence of ensembles for non-equilibrium statistical mechanical systems conjectured for fluid flows in [3, 4]. In particular, we tested the conjecture within the framework of the shell models for turbulence featuring a multiscale nonlinear dynamics.

In these systems, the issue of non-equilibrium ensemble equivalence translates into the quest for equivalence of the macroscopic dynamics between systems with different modelizations of the viscous forces. The standard choice is to use a constant viscosity, which, from the mathematical point of view, explicitly breaks the time-reversal symmetry of the equations of motion. However, given the reversibility of the microscopic dynamics, it is natural to speculate that a macroscopic description preserving such a fundamental symmetry should be possible.

Models exhibiting a time reversal symmetry can be realized by using a time-dependent viscosity designed to enforce the conservation of some observable, quadratic in the velocity via, for instance, Gauss' principle for anholonomous constraints [1, 29.
The construction of the reversible models is not unique, relying on the choice of the observable to keep constant in the time-reversible dynamics. We found that the equivalence between the two statistical ensembles holds, as expected, in the quasi-equilibrium regime, i.e. in the limit of very large Reynolds number when keeping constant the number of shells (i.e. the ultraviolet cut-off). Moreover, when the reversible model is constructed by imposing a constraint impacting preferentially the smallest and fastest scales of the system, e.g. when enforcing the conservation of enstrophy, equivalence is obtained also in the energy cascade regime, likely, owing the the robustness of the cascade mechanisms against the mechanism of energy dissipation.

The results in this study, together with similar findings on the 2D Navier-Stokes equations [ 6 and on the Lorenz '96 system [7, strengthen the case for the nonequilibrium statistical equivalence to hold also for other physically relevant non-equilibrium dynamical systems and, in particular, for the 3D Navier-Stokes equations, for which it was originally conjectured [3].

Besides the theoretical interest, the results here presented offer more freedom in modeling viscous forces in non-equilibrium systems, with particular reference to the ones of interest in fluid dynamics. Specifically, the ideas discussed in this paper could be relevant for small scale parametrization in atmosphere, ocean and climate models [30 32, and LES models [1], 12, where eddy viscosity need to be carefully tailored in order to have results compatible with DNS. Indeed some form of reversible modeling of the small-scale dynamics is already used in LES [13, 14]. 


\section{ACKNOWLEDGMENTS}

GG thanks L. Pizzochero for fruitful discussions and for suggesting Ref. [25]. LB and MDP acknowledge funding from the European Research Council under the European Union's Seventh Framework Programme, ERC Grant Agreement No 339032. LB, MC and MDP acknowledge the European COST Action MP1305 Flowing Matter. VL acknowledges the support of DFG Sftb/Transregio Project TRR181.

\section{Appendix A: Numerical integration scheme}

Equations (6)-13, neglecting the forcing term, have the structure

$$
\frac{d}{d t} u_{n}(t)=g_{n}\left[\left\{u_{n}(t)\right\}\right]-\nu k_{n}^{2} u_{n}(t),
$$

where $g_{n}\left[\left\{u_{n}(t)\right\}\right]$ stands for the non-linear term at shell $n$, calculated on the velocity configuration $\left\{u_{n}(t)\right\}$ at time $t$.

When $\nu$ is constant in time, we adopted the following modified 4-th order Runge-Kutta scheme which exactly integrates the viscous contribution:

$$
\left\{\begin{array}{l}
u_{n}(t+\delta t)=e_{n}\left\{e_{n}\left[u_{n}(t)+\frac{\delta t}{6} g_{n}\left[\left\{u_{n}(t)\right\}\right]\right]+\right. \\
\left.\quad+\frac{\delta t}{6}\left[g_{n}\left[\left\{u_{n}^{(1)}(t)\right\}\right]+g_{n}\left[\left\{u_{n}^{(2)}(t)\right\}\right]\right]+\frac{\delta t}{6} g_{n}\left[\left\{u_{n}^{(3)}(t)\right\}\right]\right\} \\
u_{n}^{(1)}(t)=e_{n}\left[u_{n}(t)+\frac{\delta t}{2} g_{n}\left[\left\{u_{n}(t)\right\}\right]\right] \\
u_{n}^{(2)}(t)=e_{n} u_{n}(t)+\frac{\delta t}{2} g_{n}\left[\left\{u_{n}^{(1)}(t)\right\}\right] \\
u_{n}^{(3)}(t)=e_{n}\left[e^{\frac{1}{2} \nu k_{n}^{2} \delta t} u_{n}(t)+\frac{\delta t}{2} g_{n}\left[\left\{u_{n}^{(2)}(t)\right\}\right]\right] \\
e_{n}=e^{\frac{1}{2} \nu k_{n}^{2} \delta t}
\end{array}\right.
$$

For the reversible models, where $\nu$ is not a constant, we introduced the following correction to the scheme:

$$
\left\{\begin{array}{l}
u_{n}(t+\delta t)=e_{n}\left\{e_{n}\left[u_{n}(t)+\frac{\delta t}{6} \hat{g}_{n}\left[\left\{u_{n}(t)\right\}\right]\right]+\right. \\
\left.\quad+\frac{\delta t}{6}\left[\hat{g}_{n}\left[\left\{u_{n}^{(1)}(t)\right\}\right]+\hat{g}_{n}\left[\left\{u_{n}^{(2)}(t)\right\}\right]\right]+\frac{\delta t}{6} \hat{g}_{n}\left[\left\{u_{n}^{(3)}(t)\right\}\right]\right\} \\
u_{n}^{(1)}(t)=e_{n}\left[u_{n}(t)+\frac{\delta t}{2} \hat{g}_{n}\left[\left\{u_{n}(t)\right\}\right]\right], \\
u_{n}^{(2)}(t)=e_{n} u_{n}(t)+\frac{\delta t}{2} \hat{g}_{n}\left[\left\{u_{n}^{(1)}(t)\right\}\right], \\
u_{n}^{(3)}(t)=e_{n}\left[e^{\frac{1}{2} \nu k_{n}^{2} \delta t} u_{n}(t)+\frac{\delta t}{2} \hat{g}_{n}\left[\left\{u_{n}^{(2)}(t)\right\}\right]\right] \\
e_{n}=e^{\frac{1}{2} \nu\left[\left\{u_{n}(t)\right\}\right] k_{n}^{2} \delta t}, \\
\hat{g}_{n}\left[\left\{u_{n}^{(i)}(t)\right\}\right]=g_{n}\left[\left\{u_{n}^{(i)}(t)\right\}\right]-\left(\nu\left[\left\{u_{n}^{(i)}(t)\right\}\right]-\right. \\
\left.\quad+\nu\left[\left\{u_{n}(t)\right\}\right]\right) k_{n}^{2} u_{n}(t) .
\end{array}\right.
$$

[1] G. P. Morriss and D. J. Evans, Statistical Mechanics of Nonequilbrium Liquids (ANU Press, 2013).

[2] Z.-S. She and E. Jackson, "Constrained euler system for navier-stokes turbulence," Phys. Rev. Lett. 70, 1255 (1993).

[3] G. Gallavotti, "Equivalence of dynamical ensembles and navier-stokes equations," Phys. Lett. A 223, 91 (1996).

[4] G Gallavotti, "Dynamical ensembles equivalence in fluid mechanics," Physica D 105, 163 (1997)

[5] L. Rondoni and E. Segre, "Fluctuations in twodimensional reversibly damped turbulence," Nonlinearity 12, 1471 (1999).

[6] G. Gallavotti, L. Rondoni, and E. Segre, "Lyapunov spectra and nonequilibrium ensembles equivalence in $2 \mathrm{~d}$ fluid mechanics," Physica D 187, 338 (2004).

[7] G. Gallavotti and V. Lucarini, "Equivalence of nonequilibrium ensembles and representation of friction in turbulent flows: The lorenz 96 model," J. Stat. Phys. 156, 1027 (2014).

[8] V.S. L'vov, E. Podivilov, A. Pomyalov, I. Procaccia, and D. Vandembroucq, "Improved shell model of turbulence," Phys. Rev. E 58, 1811 (1998)

[9] L. Biferale, "Shell models of energy cascade in turbulence," Annu. Rev. Fluid Mech. 35, 441 (2003).

[10] T. Bohr, M.H. Jensen, G. Paladin, and A. Vulpiani,
Dynamical systems approach to turbulence (Cambridge University Press, 2005).

[11] B. Galperin and S. A. Orszag, Large eddy simulation of complex engineering and geophysical flows (Cambridge University Press, 1993).

[12] P. Sagaut, Large eddy simulation for incompressible flows: an introduction (Springer Science \& Business Media, 2006).

[13] D. Carati, G.S. Winckelmans, and H. Jeanmart, "On the modelling of the subgrid-scale and filtered-scale stress tensors in large-eddy simulation," J. Fluid Mech. 441, 119 (2001).

[14] L. Fang, W.J.T. Bos, L. Shao, and J.-P. Bertoglio, "Time reversibility of navier-stokes turbulence and its implication for subgrid scale models," J. Turb. , N3 (2012).

[15] A. Vela-Martín and J. Jiménez, "Reversibility in the 3d inertial turbulent cascade," in Extended abstract presented at the Conference ETC15. 15th European Turbulent Conference, Delft, The Netherlands, 25-28 August (2015).

[16] H. Touchette, "Ensemble equivalence for general manybody systems," EPL 96, 50010 (2011).

[17] H. Touchette, "Equivalence and nonequivalence of ensembles: Thermodynamic, macrostate, and measure levels," J. Stat. Phys. 159, 987-1016 (2015). 
[18] R. H. Kraichnan, "Eddy viscosity in two and three dimensions," J. Atm. Sci. 33, 1521 (1976).

[19] F. Leyvraz and S. Ruffo, "Ensemble inequivalence in systems with long-range interactions," J. Phys. A 35, 285 (2002).

[20] G. Gallavotti and E. G. D. Cohen, "Dynamical ensembles in nonequilibrium statistical mechanics," Phys. Rev. Lett. 74, 2694 (1995).

[21] G. Gallavotti, "Reversible Anosov diffeomorphisms and large deviations." Math. Phys. Electr. J. 1, 1 (1995).

[22] G. Gallavotti, "Dynamical ensembles equivalence in fluid mechanics," Physica D 105, 163 (1997).

[23] G. Gallavotti, L. Rondoni, and E. Segre, "Lyapunov spectra and nonequilibrium ensembles equivalence in $2 \mathrm{~d}$ fluid," Physica D 187, 358 (2004).

[24] U. Frisch, Turbulence: The Legacy of A. N. Kolmogorov (Cambridge University Press, 1995).

[25] P. Constantin, B. Levant, and E. Titi, "Analytical study of shell models of turbulence," Physica D 219, 120 (2006).

[26] M. De Pietro, L. Biferale, G. Boffetta, and M. Cencini, "Time irreversibility in reversible shell models of turbu- lence," Eur. Phys. J. E 41, 48 (2018).

[27] Further simulations (not shown) performed at the best of our computational possibilities (resolutions up to $N=$ 45 and values of $\Omega$ up to $\sim 10^{12}$ ) did not show a clear trend towards such an equipartition and we consider the question still open.

[28] L. Biferale, D. Pierotti, and A. Vulpiani, "Timereversible dynamical systems for turbulence," J. Phys. A 31, 21 (1998).

[29] E. T. Whittaker, A treatise on the analytical dynamics of particles and rigid bodies (Cambridge University Press, 1988).

[30] T. N. Palmer and P. D. Williams, Stochastic Physics and Climate Modelling, edited by P. Williams and T. Palmer (Cambridge University Press, Cambridge, 2009) p. 496.

[31] C. L. E. Franzke, T. J. O'Kane, J. Berner, P. D. Williams, and V. Lucarini, "Stoch. clim. theo. model." 6, 63 (2015).

[32] J. Berner et al., "Stochastic parameterization: Toward a new view of weather and climate models," Bull. Am. Meteor. Soc. 98, 565 (2017). 